Livraisons

d'Histoire

de l'Architecture

\section{Livraisons de l'histoire de l'architecture}

$29 \mid 2015$

Ornements en architecture

\title{
La renaissance du château de Mouchy (1855-1866)
}

The Castle of Mouchy

Das Schloss von Mouchy

\section{Sophie Derrot}

\section{CpenEdition}

\section{Journals}

Édition électronique

URL : http://journals.openedition.org/lha/472

DOI : 10.4000/lha. 472

ISSN : 1960-5994

Éditeur

Association Livraisons d'histoire de l'architecture - LHA

Édition imprimée

Date de publication : 10 juin 2015

Pagination : 115-123

ISSN : $1627-4970$

Référence électronique

Sophie Derrot, «La renaissance du château de Mouchy (1855-1866) 》, Livraisons de l'histoire de l'architecture [En ligne], 29 | 2015, mis en ligne le 10 juin 2017, consulté le 19 avril 2019. URL : http:// journals.openedition.org//ha/472 ; DOI : 10.4000/lha.472

Ce document a été généré automatiquement le 19 avril 2019

Tous droits réservés à l'Association LHA 


\title{
La renaissance du château de Mouchy (1855-1866)
}

\author{
The Castle of Mouchy \\ Das Schloss von Mouchy
}

Sophie Derrot

Sis dans une ancienne baronnie de l'Oise, entre Mouy et Noailles, le château de Mouchy trouve son origine dans une place forte médiévale, incendiée une première fois par Louis le Gros au début du XII ${ }^{e}$ siècle ${ }^{1}$, puis reconstruite deux fois, la dernière au XVI ${ }^{e}$ siècle. Le domaine échoit à la famille de Noailles au milieu du XVII ${ }^{e}$ siècle; elle le conserve durant la Révolution qui mit cependant à mal le château². Philippe Louis Marc Antoine de Noailles (1752-1819), duc de Mouchy ${ }^{3}$, vient s'y installer en 1800, à son retour d'Angleterre. Le château Renaissance est agrandi dans le même style et embelli par ses descendants au cours du XIX ${ }^{\mathrm{e}}$ siècle : des écuries sont notamment construites en $1825^{4}$ et les deux grandes phases de travaux auxquelles la présente étude s'intéresse sont menées au milieu des années 1850 puis au milieu des années 1860. La commune de Mouchy-leChâtel ne compte pourtant aujourd'hui aucun château : les troupes nazies ayant occupé le bâtiment pendant la seconde guerre mondiale et l'ayant laissé en mauvais état, il sera en grande partie détruit par son propriétaire vers 1961. Ne subsistent plus aujourd'hui que la Tour carrée, donjon du XII ${ }^{e}$ siècle, et certains éléments datant du XIX siècle : le caveau de la famille, la terrasse, les écuries, l'église de Mouchy-le-Châtel, reconstruite en même temps que le château, ainsi que la chapelle, la conciergerie et le portail monumental d'entrée, situés sur la commune voisine d'Heilles (hameau de Mouchy-la-Ville).

2 Le troisième quart du XIX siècle voit donc l'apogée du domaine de Mouchy, qui doit alors se parer de tout le lustre nécessaire au prestige d'une grande famille de la noblesse fréquentant la cour de Napoléon III. En effet, les initiateurs du dernier agrandissement du château, Antoine de Noailles et sa femme, Anna Murat, sont proches du couple impérial et se rendent notamment aux séries organisées à Compiègne, non loin de Mouchy. Le château familial se trouvait en quelque sorte placé comme un parallèle Renaissance avec le gothique Pierrefonds, alors en pleine restauration par Viollet-le-Duc. Il s'agit alors de faire $\mathrm{du}$ domaine familial une grande demeure aristocratique, suivant la mode 
Renaissance, à partir d'un existant historique, afin de magnifier le passé d'un grand nom - et donc son présent. La restauration et l'embellissement du domaine sont confiés, dans les années 1850 comme 1860, à l'architecte Hippolyte-Alexandre Destailleur, qui s'adjoint le sculpteur-ornemaniste Michel Liénard. Tous deux sont très appréciés à l'époque et reconnus pour leur connaissance des styles historiques ; ils sont par ailleurs familiers des réalisations s'inscrivant dans un programme de prestige.

Hippolyte-Alexandre Destailleur (1822-1893), fils de l'architecte François Destailleur et élève d'Achille Leclère, est très actif sous le second Empire et jusqu'aux années 1890. Plus connu peut-être pour ses activités de collectionneur d'estampes et de dessins, il construit néanmoins tout au long de sa carrière plusieurs hôtels et châteaux pour la noblesse et la grande bourgeoisie, comme l'hôtel d'Haussonville (1856), ainsi que des tombeaux, dont la chapelle funéraire de Napoléon III et du prince impérial à Farnborough. Une grande partie de ses réalisations architecturales sont aujourd'hui détruites mais certaines subsistent, comme le château de Franconville, construit pour le duc de Massa, dans le Vald'Oise (1876-1882). Il trouve un collaborateur idéal en la personne de Michel Liénard $(1810-1870)^{5}$. Ce dernier, qui commence sa carrière à la fin des années 1830, est alors reconnu comme un maître du néo-Renaissance, bien que ses réalisations aient été loin de se limiter à ce style. Les contacts de Liénard avec la famille Destailleur commencent tôt : son maître, Jean-Baptiste Plantar (1790-1879), dernier sculpteur des bâtiments du Roi, avait collaboré avec François Destailleur, père d'Hippolyte, pour la construction du ministère des Finances, rue de Rivoli, en 1826, puis peut-être sur la restauration du château d'Amboise pour les Orléans. Liénard et François Destailleur travaillent ensuite ensemble en 1845-1846 à la construction du passage Jouffroy, à Paris ( $9^{\mathrm{e}}$ arr.).

4 Si le chantier de Mouchy intervient assez tôt dans la carrière d'Hippolyte Destailleur, il appartient à la dernière partie de celle de Liénard. Au moment de la première phase de travaux, dans les années 1850, Michel Liénard est au sommet de sa carrière, riche de très nombreuses collaborations dans de multiples domaines. Il bénéficie dès le début de sa carrière d'appuis dans les milieux aristocratiques qui lui permettent de se voir confier de prestigieuses commandes, telle la chapelle royale des Orléans, à Dreux (1839-1845). Avant Mouchy, l'ornemaniste a déjà travaillé sur plusieurs grandes demeures, notamment avec l'architecte Pierre-Charles Dussillon (1804-1860), comme le château de Regnière-Écluse pour le comte d'Hinnisdal (vers 1844-1845), et il a occupé peu avant une place importante dans les décors intérieurs du palais du quai d'Orsay (1852-1855). Mais le château de Mouchy est sans contexte sa plus vaste réalisation dans le domaine architectural. Une documentation assez riche permet de rendre compte de l'ampleur du chantier et de l'importance de cette commande pour le sculpteur: des mémoires de travaux couvrent la période entre 1853 et 1866, complétés de quatre lettres du milieu des années $1860^{6}$; des dessins de Liénard et Destailleur sont conservés au musée des Arts décoratifs de Paris ${ }^{7}$ et à la Kunstbibliothek de Berlin ${ }^{8}$; des détails du travail de Liénard pour le château figurent dans son recueil de gravures posthume, le Portefeuille de Liénard (Paris, C. Claesen, v.1879), (ill. 1). Le fonds Destailleur des Archives nationales comprend par ailleurs des plans en élévation avant et après les travaux ${ }^{9}$. Enfin, Pierre-Ambroise Richebourg, photographe au statut quasi-officiel sous le Second Empire, a également consacré deux campagnes photographiques au château, laissant un ensemble précieux témoignant des états successifs du bâtiment ${ }^{10}$. Grâce à l'étude de ces sources, on s'aperçoit que Liénard est chargé de tous les travaux concernant le décor extérieur et peut-être une partie de l'intérieur ${ }^{11}$. 
III. 1 : Groupe de motifs d'ornements du château de $M^{\star \star \star \star ~}$

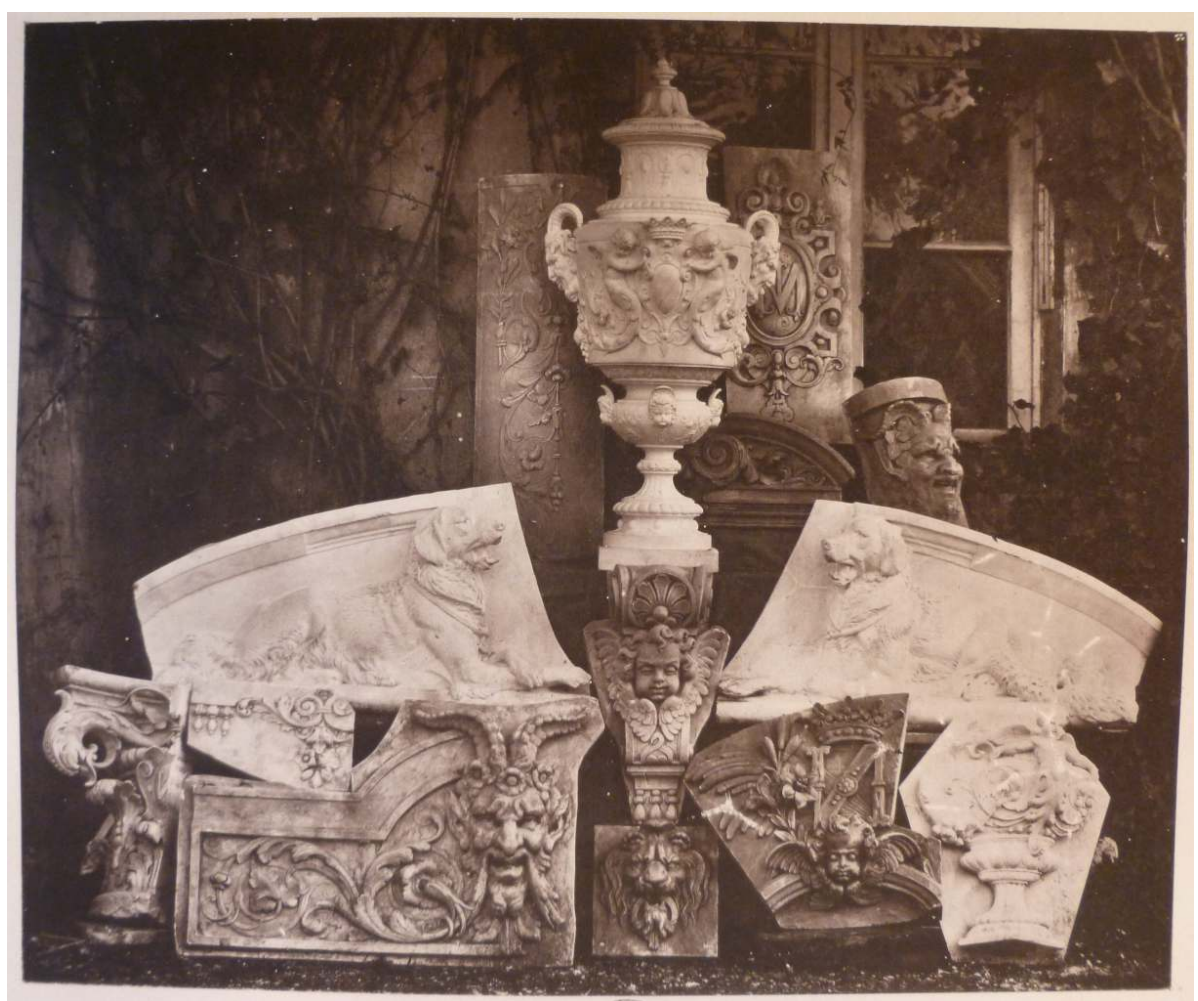

dans Portefeuille de Liénard, Paris, C. Claesen, [v. 1879], pl. 31. Coll. part.

5 Avant de restaurer le château, la famille de Noailles-Mouchy avait déjà demandé à Hippolyte Destailleur de construire vers 1852-1853 la sépulture de la famille, toute proche de l'église de Mouchy mais située à l'intérieur de la propriété ducale ${ }^{12}$, et en 1854 de commencer la restauration de la chapelle qui se trouve non loin du château. Liénard participe à ces deux chantiers, comme en attestent trois mémoires de travaux ${ }^{13}$.

Les sources permettent de reconstituer les deux temps des travaux du château au milieu $\mathrm{du}$ siècle. Certaines photographies de Richebourg représentent le bâtiment après la première phase, d'autres après la seconde. Henri de Noailles (1808-1854) commence les travaux dans les années 1840 , en faisant construire la terrasse qui existe encore aujourd'hui, du côté de la vallée ${ }^{14}$. Sa veuve, Cécile de Noailles (1812-1858) entame la restauration du bâtiment historique entre 1855 et $1860^{15}$. S'ensuivent quelques années de pause dans les travaux du château, pendant lesquelles l'église de Mouchy est restaurée par les mêmes Destailleur et Liénard, entre 1860 et 1864. Antoine de Noailles (1841-1909), fils des précédents et nouveau duc de Mouchy, se marie en 1865 à Anna Murat (1841-1924), liée à Napoléon III. À la suite de ce mariage et de la fréquentation par le couple de la cour impériale, le château est agrandi par d'une aile supplémentaire, dont l'essentiel de la construction s'achève vers 1866. Divers travaux se continueront jusqu'en 1873 et Destailleur reprendra la chapelle en $1882^{16}$.

7 Si nous ne disposons pas de plan exact de l'état du château avant les travaux, les documents iconographiques permettent de reconstituer une partie de son agencement. Le bâtiment est placé parallèlement à une hauteur et domine le parc de l'un de ses côtés. L'arrivée par la route se fait de l'autre côté, dans la cour d'honneur, où le château se présente avant 1865 sous la forme d'un L: en face du visiteur se dresse le bâtiment 
Renaissance, haut de trois étages dont le dernier est en combles, et à sa droite, en avant, la grosse tour médiévale qui sera reliée au bâtiment principal lors de la seconde phase des travaux ${ }^{17}$. Du côté qui donne sur le parc, la façade surmonte la grande terrasse, à laquelle on accède depuis le château par un petit escalier qui descend du balcon.

D'après les mémoires, la première phase des travaux concerne la remise en état du bâtiment dit «Henri II ». L'objectif annoncé -redonner au château son faste tout en respectant l'existant- est clairement perceptible dans les mémoires. Ceux-ci rappellent en cela un des premiers chantiers de Liénard, la restauration du château de Blois sous la direction de Félix Duban: d'un côté il crée et dessine lui-même une grande partie des ornements, de l'autre, il s'inspire de ce qui subsiste, comme le montrent certaines mentions des mémoires de travaux : « lucarnes copiées des anciennes », «balustres imités des anciens ", « restauration de la frise ». Les travaux de sculpture sont alors clairement désignés comme complétant la partie ancienne du château : le principe de ces premiers travaux, peut-être donné par les commanditaires, était que la structure architecturale générale devait être respectée et que chaque ouverture et pilastre devait être orné. Ce dernier point est particulièrement flagrant lorsque l'on compare les élévations dessinées par Destailleur avant et après les travaux ${ }^{18}$, on s'aperçoit alors que la remise en état dépasse la simple restauration et représente une entreprise considérable ${ }^{19}$.

La façade « Henri II » est alors sans conteste l'objet de toutes les attentions, étant celle qui doit révéler la magnificence du château au nouvel arrivant. Les tourelles octogonales encadrant la façade voient leur décoration renouvelée et enrichie. Les cinq lucarnes qui bordent la toiture s'inspirent très clairement de celles des châteaux de la Loire que Liénard et Destailleur connaissent bien, particulièrement Blois et Amboise, avec les motifs de coquilles de leur fronton et les chambranles en pilastres et les quilles qui les surmontent. Le chêneau du toit est également refait, ainsi que la crête de zinc très ornée qui le surmonte. Le luxe de la façade se révèle surtout dans l'ornementation des deux premiers étages et dans le porche d'entrée. Sous la corniche du toit et sous celle du premier étage courent deux frises différentes subsistant du château du XIX ${ }^{\mathrm{e}}$ siècle : l'une est composée de rinceaux et l'autre alterne rosaces et bucranes, dans un esprit plus proche de la première moitié du XVII ${ }^{e}$ siècle que de la Renaissance d'Henri II, mais restaurée par Liénard selon ses motifs originaux et non remplacée par une frise plus en harmonie avec le reste de l'ornementation. Les initiales de la famille propriétaire ( $\mathrm{N}$ de Noailles et $\mathrm{M}$ de Mouchy entrelacés) sont très présentes dans la décoration du château, prenant place dans les frontons au milieu de rinceaux, dans les chapiteaux des pilastres, ou dans les frises ici et là. Elles sont la plupart du temps accompagnées de la couronne ducale et de branches de laurier ou d'olivier, comme sur les pilastres entourant les fenêtres du premier étage. Cette omniprésence des symboles des propriétaires rappelle classiquement les salamandres de François $\mathrm{I}^{\text {er }}$ et les porcs-épics de Louis XII sur les châteaux de Blois ou de Chambord.

10 Entre les fenêtres du premier étage prennent place cinq grands trophées sculptés, soutenus par des agrafes et terminés par de grands lambrequins. Ces trophées ont été dessinés et réalisés par Liénard et suivent chacun un thème différent, tournant autour des plaisirs aristocratiques : les arts, la chasse, les festins, la guerre, les jeux et la musique. Ces trophées représentent une étape importante du travail de Liénard au château de Mouchy : les œuvres semblables qu'il avait pu produire auparavant n'avaient pas eu cette ampleur et cette place centrale dans la décoration d'un bâtiment. Ces trophées seront photographiés, gravés et publiés ${ }^{20}$. Au premier étage, entre les fenêtres, se trouvent de 
grands cadres sculptés avec autant de minutie, ornés d'une fleur de lys dans un fronton, de consoles fleuronnées, d'un cartouche, de moulures, contenant un socle destiné à supporter un buste ${ }^{21}$. Ces socles - de grands culs de lampe en crédence ornés de blasons, de couronnes, de fleurons et de têtes de lion - doivent mettre en valeur les bustes représentant chacun un roi de France sous lequel les Noailles se sont illustrés ${ }^{22}$. Les initiales de ces souverains se retrouvent dans les motifs qui ornent les angles des cadres, sous la forme de monogrammes.

11 La dernière pièce majeure de cette première restauration est le porche d'entrée, la voie d'honneur pour rentrer dans le château. Ce porche est créé au moment des travaux et Liénard en sculpte tous les détails d'ornementation, extérieurs comme intérieurs. L'entrée est dramatisée par son encadrement: de chaque côté de la porte, deux colonnes cannelées, ornées de bracelets et de brindilles, entourent une niche où se trouve un grand vase en bronze surmonté d'un cartouche de marbre de couleur et d'un $\mathrm{N}$ couronné. Le visiteur entre ensuite à l'intérieur du porche, lui aussi rythmé par des colonnes et des pilastres aux chapiteaux semblables, sous une voussure décorée de rosaces et de têtes de satyres, avant d'arriver à la porte surmontée par deux chiens de chasse, gueule ouverte, allongés et attachés de part et d'autre d'un écusson couronné portant blason ${ }^{23}$.

Cette phase de restauration s'apparente à de la reconstruction. Si les murs et quelques ornements subsistent, le décor semble avoir été suffisant pour pouvoir s'en inspirer, mais pas assez pour cadrer avec le château tel que le souhaitent les ducs de Mouchy. La majeure partie des sculptures d'ornement sont donc créées ou recréées et les photographies témoignent de la réussite de l'entreprise. Il ne s'agit pas ici d'une restauration aussi stricte que celle de Blois, mais plutôt une remise au goût du jour dans le style de la Renaissance tardive.

Environ cinq ans après la fin des travaux sur les parties anciennes, le nouveau duc de Mouchy décide de faire agrandir le bâtiment. Il est peu probable ce projet existait dès le début de la précédente campagne de restauration. En effet, une partie des réalisations de la fin des années 1850 est détruite par les nouveaux aménagements. L'agrandissement du château se fait par le rajout d'une aile partant perpendiculairement à l'aile Renaissance et faisant face à la route, et par le rattachement de la tour médiévale au château. Le bâtiment au complet prend donc désormais la forme générale d'un $\mathrm{U}^{24}$. Nous disposons de beaucoup moins de témoignages iconographiques sur cette nouvelle partie, mais le dernier mémoire de travaux de Liénard concerne entièrement ce chantier ; il montre que le travail de l'atelier de Liénard est là encore important ${ }^{25}$ et qu'il a englobé tout le nouveau bâtiment, y compris les modifications de la partie ancienne, comme la serre donnant sur la salle à manger de l'aile Henri II, ou la reprise des chêneaux de celle-ci. Sur l'aile XIX ${ }^{e}$ siècle, Liénard suit la ligne dictée par le reste du château, avec les mêmes motifs dominants : culs de lampe, rosaces, dauphins, coquilles, moulures d'oves, de cordes ou de rubans, et bien sûr les lettres $\mathrm{N}$ et $\mathrm{M}$ sous de multiples formes (gravées, sculptées, en fronton, en frise, dans des cartouches, etc.). Il sculpte aussi des trophées et des cadres pour des bustes en rappel de ceux de l'aile Henri II. La pièce maîtresse de ce chantier est la grande tour d'Horloge : construite à l'extrémité de l'aile Renaissance, en pendant de la tour qui se trouve au coin des ailes anciennes, elle est pareillement surmontée d'un toit conique très haut, garni des quatre lucarnes portant les horloges qui lui donnent son nom. Ces horloges, réalisées par Liénard, sont ornées d'un grand cartouche, de guirlandes de fruits, d'une tête d'Apollon, de hiboux, de coqs, avec des moulures de godrons autour du cadran. Une corniche en créneaux tient lieu de rappel de l'architecture la plus 
ancienne. Le reste de l'aile semble dans la même ligne esthétique que l'aile Renaissance, avec deux étages, puis un étage de combles ornés de lucarnes, alternant des grands et des petits modèles. Les combles de la partie ancienne sont encore enrichis pour s'harmoniser avec la nouvelle construction. La nouvelle aile porte elle aussi sur son toit de grandes cheminées aux armes des ducs de Mouchy, rappelant celles du palais du Louvre.

Si les mémoires de travaux nous donnent une idée de l'importance du rôle de Liénard dans la décoration du château de Mouchy, les photographies nous permettent de visualiser une grande demeure aristocratique de style Renaissance repris au XIX ${ }^{e}$ siècle. Les rappels des architectures $d u X V I^{e}$ siècle sont bien visibles et le respect du décor subsistant a sans doute contribué à une certaine unité dans la décoration. Le style adopté s'inspire certes des éléments historiques subsistants, mais s'inscrit dans une Renaissance telle qu'elle est vue au milieu du XIX siècle, très large, allant de la fin du gothique flamboyant à Louis XIII. Les éléments redondants et leur dimension nobiliaire servent le message qu'un décor omniprésent doit faire passer. L'action de Destailleur et Liénard ne se limite pas au château Renaissance : l'église du village est quant à elle restaurée suivant son époque de construction, dans un gothique très simple, avec un grand tympan roman. Les différentes réalisations suivent un plan complet de réaménagement du domaine, soucieux de dégager une relative cohérence entre ses différents éléments tout en maintenant les traces de l'historique de chacun des bâtiments.

Si la manière de travailler de Félix Duban, sur le chantier de Blois notamment, est bien connue, celle d'Hippolyte Destailleur n'a jamais encore fait l'objet d'une étude publiée. Il est difficile d'estimer quelle a été la marge de manœuvre du sculpteur par rapport à l'architecte sur ce chantier et de déterminer d'où vient la cohérence historique du décor. Destailleur collectionne déjà les dessins topographiques et les gravures d'architecture qui feront de lui un connaisseur averti des réalisations historiques. Cependant, Liénard peut aussi exploiter sa formation avec Jean-Baptiste Plantar sur les chantiers des châteaux de la Couronne et ses premières expériences sous la direction de Félix Duban, l'un des pères de l'architecture néo-Renaissance française. En engageant cet artiste, Destailleur était certain d'avoir recours à un sculpteur non seulement capable de travailler sur une base historique, mais également connu pour sa capacité à inventer de nouveaux ornements. Au moment du chantier de Mouchy, Liénard est probablement à la tête d'un grand atelier qui lui permet de répondre à une commande de cette ampleur, mais qui reste encore assez mal connu. Outre celui du sculpteur Moreau ${ }^{26}$, un nom se détache dans les lettres et les mémoires de travaux qui subsistent, celui d'Alfred Doussamy (1831-1885), gendre de Liénard. Le chantier de Mouchy tient en effet lieu de passation entre les deux hommes, même si Doussamy ne reprend pas formellement l'atelier de Liénard après la mort de ce dernier, en 1870. Doussamy travaille par la suite à plusieurs reprises avec Hippolyte Destailleur, notamment pour l'hôtel de Mouchy à Paris, situé sur le boulevard de Courcelles et également rénové par l'architecte ${ }^{27}$, mais détruit en 1958.

Surtout, le jeune sculpteur collabore avec Destailleur sur le pendant anglais de Mouchy, Waddesdon Manor, construit pour le baron Ferdinand de Rothschild entre 1874 et $1899^{28}$. Grand amateur d'objets d'art Renaissance, le baron avait été frappé par les châteaux français de Touraine et embauche sur le souvenir de Mouchy Hippolyte Destailleur, puis son fils, Walter-André d'Estailleur (1867-1940) pour construire son domaine au nordouest de Londres. Les deux constructions offrent de nombreux points de similarités et, si Waddesdon Manor ne connaît pas de base ancienne, il s'inspire de plusieurs châteaux français (Chambord, Maintenon, Chaumont-sur-Loire, etc.). À côté de ces influences 
historiques, de nombreux ornements sont repris à l'identique de Mouchy pour décorer Waddesdon, comme l'indiquent des annotations sur certains dessins du fonds Destailleur de Berlin ${ }^{29}$. Le travail de Doussamy est alors précieux : il arrive à Waddesdon en $1879^{30}$ et tient lieu de véritable trait d'union entre les deux chantiers, au point que son nom apparaît au côté de celui de l'architecte sur l'œil de bœuf du pavillon central.

\section{NOTES}

1. Antoine-Joseph Warmé, Mouy et ses environs: Angy, Bury, Ansacq, Cambronne, Heilles, Hondainville, Neuilly, Mello, Mouchy-le-Chatel, Saint-Félix, Thury-sous-Clermont, Ully-Saint-Georges, Beauvais, D. Père, 1873, t. VI-513 p.

2. Anonyme, "Château de Mouchy (département de l'Oise)", dans Le Magasin pittoresque, t. XXXIII, 1865, p. 245-246, ill.

3. Le titre de duc de Mouchy est porté par la branche cadette de la famille de Noailles, à partir du milieu du XVIIIe siècle.

4. Notice sur Mouchy-le-Châtel, Beauvais, Moniteur de l'Oise, 1893, p. 9.

5. À propos de cet artiste, voir Sophe Derrot, Michel Linéard (1810-1870), luxuriance et modestie de l'ornement au XIXe siècle, thèse de doctorat soutenue en 2014, École des hautes études, vol. 1 (391 p.), vol. 2 (238 p.).

6. Ces mémoires et lettres sont conservées par l'actuel duc de Mouchy, que je remercie pour son aide. Treize mémoires concernent les réalisations de Liénard et permettent d'estimer les sommes considérables qu'il a touchées pour ce travail, qui se montent au total à plus de 174895 francs.

7. Une quinzaine de dessins issus des fonds Linéard et Prignot conservés au département des Arts graphiques du musée des Arts décoratifs ont pu être reliés à Mouchy.

8. Les albums Destailleur conservés par la Kunstbibliothek de Berlin comptent un grand nombre de dessins en rapport avec Mouchy, souvent daté, parfois annotés mais jamais signés. La plupart de ces dessins semblent se rapporter à la seconde phase des travaux extérieurs, ainsi qu'aux travaux intérieurs.

9. Arch. nat., fonds Destailleur, 536 AP. Ce fonds, conservé aujourd'hui sur le site de Fontainebleau, n'est pas accessible pour le moment.

10. Vingt-sept de ces photographies se trouvent aujourd'hui dans le fonds Destailleur de l'Académie d'Architecture (nos 522.1 à .22) et dans le fonds Richebourg du département des Estampes de la Bibliothèque nationale de France (EO-28 (1 et 2) - Fol et Pet. Fol.). D'autres photographies, prises vers 1955 puis au moment de la démolition en 1961, se trouvent dans le fonds Fernand Watteuw, conservé aux archives départementales de l'Oise (30 Fi 7/438 à 440, 30 Fi 2/611 à 615 et 30 Fi 15/1014/1 à 1016/1 et 4959/1).

11. Concernant l'intérieur, il n'y a qu'une mention dans les mémoires qui laisserait penser que Liénard y a aussi œuvré, à propos de « balustres ou vases pour le haut du lit de 
Madame la duchesse de Mouchy ». On conserve par ailleurs les noms de sculpteurs sur bois qui ont probablement travaillé sur les décors intérieurs, comme les frères Huber.

12. Des plans pour ce caveau se trouvent dans les albums Destailleur de la Kunstbibliothek de Berlin.

13. Ces mémoires concernent des travaux de sculptures pour le caveau et sa porte, ainsi qu'une sculpture pour la chapelle.

14. Notice sur le Mouchy-le-Châtel, op. cit., p. 10.

15. Le Magasin pittoresque de 1865 (art. cité) indique 1856 comme date de début des travaux. Mais les premiers mémoires de Liénard concernant le château datent bien de 1855.

16. Des plans de cette chapelle, datés de 1882 , se trouvent dans le fonds des Arch. nat.

17. Notices sur Mouchy-le-Châtel, op.cit., p. 10.

18. Ces élévations, conservées dans les fonds des Archives nationales, sont reproduites dans Bruno Pons, Waddesdon Manor, Architecture and Panelling, Londres, P. Wilson for the Waddesdon trust, 1996, p. 66.

19. L'ampleur des travaux se perçoit également dans la somme que l'atelier Liénard touche pour cette première phase : 136 595,18 francs pour le cumul des mémoires de 1855 à 1860 .

20. Ceux des arts et de la guerre ont été gravés dans le dernier des recueils des travaux de Liénard, le Portefeuille de Liénard et tous sont photographiés, tout comme les modèles des trophées de la chasse et des festins, dont les photographies sont signées par Liénard (photographies nos 20 et 21 du fonds Destailleur, Académie d'Architecture).

21. Un calque pour le cadre pour le buste de François Ier se trouve dans le fonds Destailleur de la Kunstbibliothek de Berlin.

22. L'auteur de ces bustes et leur date de réalisation sont inconnus. Les mémoires de Liénard mentionnent François Ier, Henri II (illustration de ce cadre dans la collection Maciet de la bibliothèque des Arts décoratifs, album 233, 12), Henri IV, Louis XIII et Louis XIV.

23. Plusieurs dessins du fonds Liénard du musée des arts décoratifs présentent des études pour ce porche, notamment pour les chapiteaux des colonnes et pour les panneaux de la porte.

24. Un plan du château en novembre 1865 se trouve dans le fonds Destailleur des Arch. nat.

25. La somme touchée s'élève à 38300 francs, dont dix-sept voyages de sculpteur.

26. Ce sculpteur est mal connu, mais il est cité à plusieurs reprises comme appartenant à l'atelier de Liénard. Peut-être a-t-il un lien avec le sculpteur Mathurin Moreau (1821-1912), qui collabore avec l'ornemaniste sur des fontaines pour les fonderies du Val d'Osne. À Mouchy lui sont attribués les satyres qui surplombent le porche d'entrée du château et les deux cariatides du portail monumental du domaine.

27. La revue Paris architecte de 1865 évoque plusieurs collaborations pour le duc de Mouchy entre Doussamy sculpteur et Destailleur architecte et dessinateur: un meuble pour un coffret en cristal de roche du xvie siècle (mai 1865, p. 20, pl. X), ainsi qu'une porte d'écuries (février 1867, p. 16 et pl. VII). Le motif de cette porte se retrouve dans le fonds Liénard du musée des Arts décoratifs (CD 413964 et CD 414055); d'autres dessins en lien avec l'hôtel de Mouchy se trouvent dans le fonds Prignot du même musée. Une autre 
mention de collaboration, pour une «maison avenue de l'Opéra » figure dans un album Maciet de la BAD $(242,1)$.

28. Sur Waddesdon Manor, voir B. Pons, Waddesdon Manor..., op. cit.

29. Certains dessins du fonds Destailleur de la Kunstbibliothek de Berlin concernent Waddesdon Manor, mais portent des mentions telles que «les profils sont ceux des lucarnes de Mouchy ».

30. B. Pons, Waddesdon Manor..., op.cit., p. 69.

\section{RÉSUMÉS}

Tout proche de la résidence royale puis impériale de Compiègne, le château de Mouchy (Oise), ainsi que son domaine, est embelli et agrandi au XIX ${ }^{\mathrm{e}}$ siècle, sur la volonté de la famille de Noailles. Au milieu du siècle, Henri de Noailles puis son fils, Antoine, ducs successifs de Mouchy, confient le chantier au jeune architecte Hippolyte Destailleur. Celui-ci emploie le sculpteurornemaniste Michel Liénard et son atelier, pour le seconder sur ce chantier qui mêle restauration et construction. La seconde moitié du siècle voit se succéder deux grandes phases de travaux (1855-1860 puis 1860-1864) qui visent à donner au château historique de la Renaissance le prestige d'une grande demeure nobiliaire. Fins connaisseurs du style Renaissance, Destailleur et Liénard recréent un décor sculpté à l'aulne de cette volonté de faste, en s'inspirant de modèles historiques et du rayonnement de la famille commanditaire. Le chantier de Mouchy prend une place toute particulière dans la carrière des deux hommes. Pour Liénard, il s'agit de la dernière pierre à une carrière couronnée par le succès et la reconnaissance ; pour Destailleur, Mouchy signe des débuts flamboyants dans le domaine de la construction nobiliaire. Aujourd'hui détruit, le château de Mouchy a laissé des traces dans un autre bâtiment, Waddesdon Manor, cousin anglais également construit par Destailleur et le successeur de Liénard, Alfred Doussamy, pour le baron Ferdinand de Rothschild.

Close to the royal and then imperial residence of Compiègne, Mouchy Castle (Oise) and its estate were embellished and extended during the nineteenth century by the will of the Noailles family. In the middle of the century, Henri de Noailles and then his son, Antoine, successive dukes of Mouchy, entrusted the construction work to the young architect Hippolyte Destailleur. In order to assist him with this work which combined restoration and new construction, Destailleur employed the sculptor and ornemanist Michel Liénard and his team. During the second half of the century, between 1855 and 1866, two important phases of construction were carried out to bring back some noble prestige into the historical castle from the Renaissance. Astute experts of the Renaissance style, Destailleur and Liénard recreated a sculpted decoration commensurate to the will of splendor, and took inspiration from historical models as well from the influence of their sponsor family. The work at Mouchy Castle occupied a particular place within the career of both artists. For Liénard, it was the last part of a career crowned by success and recognition; for Destailleur, Mouchy was the blazing beginning of his work in the field of construction for nobility. The castle of Mouchy does not exist anymore, but its influence remains visible in its English cousin, Waddesdon Manor, which was also built by Destailleur and the successor of Liénard, Alfred Doussamy, for the baron Ferdinand de Rothschild. 
Das in unmittelbarer Nähe zum königlichen, später kaiserlichen Schloss von Compiègne gelegene Château de Mouchy (Departement Oise) wird zusammen mit seinem umliegenden Besitzungen im Laufe des 19. Jahrhunderts auf Veranlassung der Familie von Noailles umfassend ausgebaut und ausgeschmückt. Um die Mitte des Jahrhunderts übertragen die Herzöge von Mouchy, Henri von Noailles und später sein Sohn Antoine, die Arbeiten dem jungen Architekten Hippolyte Destailleur, der bei diesem Bauvorhaben, bei dem sich Restaurierung und Neubau mischen, vom Bildhauer und Stuckateur Michel Liénard und dessen Werkstatt unterstützt wird. Während zweier bedeutender Bauphasen in der zweiten Hälfte des 19. Jahrhunderts (1855-1860 sowie 1860-1864) soll das Renaissance-Schloss in eine prunkvolle zeitgemäße Adelsresidenz verwandelt werden. Als profunde Kenner der Renaissancekunst entwerfen Destailleur und Liénard ein aufwendig gestaltetes Dekor aus Erlenholz, das sowohl den historischen Vorbildern als auch der gesellschaftlichen Stellung der Auftraggeber Rechnung trägt. Für Liénard als auch für Destailleur bedeuten die Arbeiten in Mouchy dabei den Grundstein für eine erfolgreiche spätere Karriere, die letzterer insbesondere mit adeligen Bauaufträgen fortsetzt. Auf das heute zerstörte Schloss von Mouchy lässt sich noch anhand eines ihm eng verwandten Baus in England schließen, des Waddesdon Manor, das ebenfalls von Destailleur zusammen mit dem Nachfolger von Liénard, Alfred Doussamy, für den Baron Ferdinand von Rothschild errichtet worden ist.

\section{AUTEUR}

\section{SOPHIE DERROT}

Sophie Derrot est née en 1983 et est conservatrice à la Bibliothèque nationale de France. Archiviste-paléographe (promotion 2008), elle a soutenu en septembre 2014 une thèse de doctorat sur le sculpteur-ornemaniste Michel Liénard (1810-1870), à l'École pratique des hautes études sous la direction de Jean-Michel Leniaud. Adresse électronique : sderrot@gmail.com 